\title{
Incorporación del enfoque psicoeducativo a la gestión educativa en programas de acogimiento residencial haciendo uso de la Metodología Investigación Acción*
}

\author{
LIDIA CANQUIL SILVA** \\ Residencia Familiar Angelmo - Chile \\ MARINA ALARCÓN ESPINOZA**** \\ Universidad de La Frontera - Chile \\ ALBA ZAMBRANO CONSTANZO \\ Universidad de La Frontera - Chile
}

Recibido el 05-09-2017; primera evaluación el 07-01-2019; segunda evaluación el 25-01-2019; tercera evaluación el 23-02-2019, aceptado el 4-01-2019

\section{Resumen}

A partir de una investigación acción, se busca identificar los elementos necesarios para incorporar el enfoque psicoeducativo a la gestión educativa de una residencia familiar en el sur de Chile. Se emplearon para la producción de datos, las técnicas cualitativas de entrevistas y taller diagnóstico participativo. Los resultados refieren que componentes del enfoque psicoeducativo tales como, su comprensión de la relación ayuda, las estrategias para utilizar la vivencia compartida y las herramientas de diagnóstico, planificación y evaluación, influyen positivamente en la gestión

\footnotetext{
* Esta investigación se enmarca en un proceso de formación del Magíster y Especialización en Psicología Comunitaria, Departamento de Psicología de la Universidad de La Frontera.

** Asistente social por la Universidad de Los Lagos y magíster en Psicología Comunitaria por la Universidad de La Frontera. Asistente social en Residencia Familiar Angelmo. Correo: lidia_ canquil_2@hotmail.com

${ }^{* * *}$ Autora de correspondencia. Psicóloga en programas dirigidos a nińos, adolescentes y familias en contextos de vulnerabilidad. Terapeuta familiar Instituto Chileno de Terapia Familiar, magíster en Desarrollo Humano a escala Local y Regional por la Universidad de La Frontera. Actualmente adscrita como académico del Departamento de Psicología, Universidad de La Frontera. Cursa el Programa de Doctorado Conjunto Universidad Autónoma de Barcelona - Universidad de Barcelona en Psicología de la Comunicación y Cambio. Correo: marina.alarcon@ufrontera.cl

${ }^{* * * *}$ Psicóloga por la Universidad de La Frontera, magíster en Ciencias Sociales Aplicadas por la Universidad de La Frontera, magíster en Ciencias de la Educación Mención Desarrollo Social por la Universidad Paris XII, doctora en Psicología Social por la Universidad de Barcelona, académico del Departamento de Psicología de la Universidad de La Frontera. Correo: alba.zambrano@ufrontera.cl
} 
educativa del centro. Su incorporación requiere de un proceso sistemático y permanente en el tiempo, así como también que confluyan la presencia de criterios y condiciones para su apropiación como enfoque y metodología de intervención por parte del personal del centro.

Palabras clave: educación social, derechos del niño, educación integral, ambiente educativo, cambio institucional.

\section{Incorporation of the Psychoeducational Approach to Educational Management in Residential Care Programs using the Action Research Methodology}

\section{Abstract}

From an action research it is sought to identify the necessary elements to incorporate the psychoeducational approach to the educational management of a Family Residence in the south of Chile. The qualitative techniques of interviews and participatory diagnostic workshop were used to produce data. The results refer to components of the psychoeducational approach such as, their understanding of the relationship helps, the strategies to use the shared experience and the tools of diagnosis, planning and evaluation, positively influence the educational management of the center. Its incorporation requires a systematic and permanent process over time, as well as the confluence of the presence of criteria and conditions for its appropriation as an approach and intervention methodology by the center's staff.

Keywords: social education, rights of the child, integral education, educational environment, institutional change.

\section{Incorporaçáo da abordagem psicoeducativa à gestáo educacional em programas de assistência residencial utilizando a metodologia de pesquisa-açáo Resumo}

A partir de uma pesquisa-ação, procura-se identificar os elementos necessários para incorporar a abordagem psicoeducacional à gestáo educacional de uma Residência de Família no sul do Chile. As técnicas qualitativas de entrevistas e oficinas de diagnóstico participativo foram usadas para produzir dados. Os resultados referem-se a componentes da abordagem psicoeducativa, tais como, a compreensão do relacionamento ajuda, as estratégias para usar a experiência compartilhada e as ferramentas de diagnóstico, planejamento e avaliação, influenciam positivamente a gestão educacional do centro. Sua incorporação requer um processo sistemático e permanente ao longo do tempo, bem como a confluência da presença de critérios e condiçóes para sua apropriação como metodologia de abordagem e intervenção pela equipe do centro.

Palavras-chave: educação social, direitos da criança, educaçáo integral, ambiente educacional, mudança institucional. 


\section{INTRODUCCIÓN}

El programa de protección residencial, o centro residencial, es una medida que ha estado presente en la asistencia a los niños en situación de abandono y desprotección durante muchos siglos, con diversas denominaciones, como orfanatos, hogares de menores, reformatorios, hospicios, entre otros. Medida que buscaba dar una respuesta única y universal a la infancia desprotegida, atendiendo a sus necesidades más elementales en sustitución del ambiente familiar (Bravo y Del Valle, 2009a).

Los programas de protección residencial han tenido una evolución desde el enfoque de la irregularidad social al actual paradigma centrado en las personas menores de 18 años como sujetos de derechos (Martínez, 2010). En este contexto, los centros residenciales han tenido transformaciones y modificaciones en sus prácticas, poniendo énfasis en la intervención familiar, estándares mínimos de calidad y la incorporación de enfoques transversales de intervención, tales como: género, interculturalidad y necesidades especiales; entendiendo las medidas de internación como excepcionales y transitorias (Farías, 2010).

La residencia, en Chile, cuenta con diferentes modalidades de intervención y se clasifican en residencias especializadas en la atención de lactantes y preescolares, para mayores, transitorias de diagnósticos y especializadas en temáticas complejas. Además, se caracterizan de acuerdo a su cobertura o plazas, siendo en pequeńa, baja y media cobertura, con el objetivo de propiciar condiciones de vida familiar (Farías, 2010).

Si bien las residencias han tenido una evolución en el plano teórico y metodológico de la intervención, investigaciones desarrolladas tanto a nivel internacional como nacional dan cuenta sobre la crisis de estos programas en los últimos ańos. A nivel internacional, importantes cambios en la estructura y objetivos de esta línea programática están asociados a problemas emergentes como la llegada de menores extranjeros no acompańados, el incremento de los casos de violencia familiar ejercida por los hijos, la necesidad de atención terapéutica a niños con problemas emocionales y de salud mental, entre otros (Bravo y Del Valle, 2009b). A nivel nacional, la crisis del sistema residencial está asociada a la falta de recursos para brindar una atención adecuada a las necesidades de los niños (Guzmán, 2010). Al respecto, Martínez (2010) señala que el perfil de caso se ha complejizado y las residencias no están suficientemente preparadas para brindar una atención adecuada a quienes requieran la medida; generándose un desajuste entre la actual capacidad de dichas instituciones y los requerimientos planteados por el nuevo perfil del usuario. 
El actual escenario que deben enfrentar los centros residenciales, dadas las características y necesidades que presentan las niñas/os y adolescentes, la aparición de nuevos perfiles y nuevas problemáticas, ha puesto en cuestión la idoneidad de las residencias. Sin embargo, existe consenso en que los centros residenciales están plenamente justificados en la medida que responden a una necesidad vigente en la sociedad (Bravo y Del Valle, 2009a; Martínez, 2010).

En el contexto chileno, la intervención residencial trabaja en base a un Plan de Intervención Individual (PII), diseñado por profesionales de cada centro, orientado a la restitución de derechos vulnerados poniendo énfasis al derecho a vivir en familia en un corto o mediano plazo. Si bien el concepto PII se ha ido compartiendo con los educadores de trato directo - personas encargadas en la cotidianidad de las y los niños y adolescentes-, estos por lo general, no tienen una participación activa en la construcción del instrumento, ni en la elaboración de los objetivos a trabajar con cada niño(a) o joven (Del-Valle, 2008). Este personal se centra, más bien, en cuidados generales de los niños y niñas más que en una labor educativa intencionada sobre determinados objetivos de desarrollo de la población que atiende. Por ello, Martínez (2010), a partir de un estudio realizado en Chile, constata que en este sistema de atención no se ha considerado el potencial educativo de la comunidad que se configura al interior de los centros, ni se ha adoptado un modelo de intervención que la potencie e incorpore en el proceso a los educadores/as de trato directo, cumpliendo estos solo una función administrativa de la vida cotidiana del centro.

Por tanto, es posible señalar que las Residencias tienen actualmente el desafío de mejorar su gestión educativa, a través de mecanismos y procesos que les permitan optimizar los resultados o productos educativos de la intervención residencial. De esta forma, deben avanzar en constituirse en alternativa formativa que ofrezca un programa de atención individualizado a partir de una organización que promueva y/o estructure procesos personales para resolver positivamente necesidades del desarrollo de niño/as y abordar los eventuales impactos de la vulneración de derechos (Del-Valle, 2008).

En esta lógica, los centros residenciales tienen el desafío de constituirse en espacios de convivencia educativa cualificada, de estilo participativo y democrático, donde el personal educativo y la población acogida sean protagonistas de la acción educativa (Cruz, 2011).

A nivel internacional, se utiliza el sistema de evaluación y registro en acogimiento residencial, planteando un modelo de trabajo sistemático para la evaluación de necesidades del niño/a y la elaboración de un proyecto educativo individual que pueda ser evaluado y contrastado (Del-Valle, 2008). 
La aplicación del instrumento se ejecuta en diferentes fases: evaluación, programación, intervención y valoración del caso. Durante el proceso, se debe considerar los factores protectores y necesidades de los nińos/as y adolescentes de acuerdo a su etapa evolutiva, favoreciendo una intervención diferenciada de cada caso (Lázaro, 2009).

El contar las residencias con un proyecto educativo permite tener una evaluación diagnóstica auténtica de cada niño/a, ajustada a sus necesidades cognitivas, intelectuales, afectivas y emocionales que permiten desarrollar con eficacia la labor educativa; minimizando la improvisación y con ello evitando la victimización e invisibilización de los niños/as y adolescentes (Del-SolFlores, 2012).

A nivel nacional, también se han desarrollado acciones que permiten potenciar la capacidad educativa de las residencias, construyendo una matriz evaluativa elaborada con componentes, dimensiones y variables que permite situar en qué punto del continuo de cada variable se sitúa la residencia, posibilitando calcular un índice que denotará el nivel de capacidad socioeducativa del centro (Martínez, 2010). Del mismo modo, se propone una gestión comunitaria planificada con fines educativos, donde se visualice a la comunidad interna (residencia) como un espacio y una herramienta educativa; es decir, una pieza clave asociada estratégicamente a todas las otras intervenciones (familiar, terapéutica, en red, entre otras) en un modelo integral de intervención.

Un equipo del Departamento de Psicología de la Universidad de la Frontera y el equipo del Departamento de Psicología y Psicoeducación de la Universidad de Quebec, en un convenio de colaboración, impulsaron la incorporación de modelos de intervención psicoeducativos que potencian la capacidad educativa y preventiva de los programas que trabajan con población infantojuvenil con dificultades en adaptación psicosocial, inicialmente en la región de la Araucanía para luego extenderse a otras zonas del país (Vizcarra y Dionne, 2008).

La perspectiva psicoeducativa es un modelo integral que consta de una base filosófica, teórica y práctica que permite involucrar a todos los actores (profesionales, educadores, personal de servicio, niños y familias) en el proceso de atención al sujeto, optimizando el desempeño personal a través del fortalecimiento de sus competencias. Así también, reconoce la diversidad de las personas considerando sus contextos socioculturales, propiciando que cada persona configure su propio escenario. Igualmente, propone una metodología de trabajo individual y grupal que favorece la construcción de conocimiento, considerando en dichos espacios de encuentro el respeto por la diversidad de puntos de vista y comportamientos alternativos, ya que constan de opera- 
ciones profesionales y esquemas relacionales, previamente definidos. Así mismo, pone énfasis en la vivencia compartida que se crea en los espacios de intervención (programas), donde se generan conocimientos y estrategias necesarias para propiciar la adaptación de la persona (Vizcarra y Dionne, 2008).

La incorporación de un modelo de intervención psicoeducativo en programas de infancia, requiere de procesos que de acuerdo a la experiencia desarrollada en el proyecto para los jóvenes con dificultad en la Araucanía (PJDA), demandan de un primer momento de socialización que permita aclarar algunos requerimientos en terreno acerca de las condiciones para la efectiva implementación de una nueva forma de trabajo (Demers y Zambrano, 2006). Luego de lo cual es necesario una formación en el enfoque, puesto que el modelo de trabajo es riguroso, integral, enfocado en las fortalezas y necesidades de desarrollo del sujeto de atención (Paes-Gálvez, 2008).

La formación en el enfoque psicoeducativo demanda resaltar elementos como la utilización de vivencia compartida (hacer con el otro en la vida cotidiana), a fin de propiciar las condiciones para que el sujeto establezca vínculos entre los distintos planos de su vida: afectivo, fisiológico, cognitivo, social, moral, entre otros (Demers y Zambrano, 2008), a través de diferentes momentos educativos.

La incorporación del enfoque implica conocer e integrar un modelo filosófico y técnico por parte de todos los integrantes del equipo, lo que supone un nivel intensivo de formación. También, demanda de un nivel mayor de organización de las personas involucradas en el proceso, con miras a lograr eficiencia y una alternativa real de mejoramiento de procesos. Igualmente, permite contar con un instrumento de construcción de un diagnóstico integral del caso que se va enriqueciendo con el tiempo, incluyendo coordinadamente las miradas e informaciones provenientes de todos los agentes vinculados en la intervención indirecta y con el entorno natural del joven; posibilitando el intercambio de visiones, el desarrollo de consensos y contrastes respecto de la complejidad de su vida para configurar un sistema de fortalezas, debilidades, intereses y necesidades del joven (Colque y Chesta, 2006).

Otros autores hacen referencia a elementos contextuales que influyen en la incorporación del enfoque psicoeducativo estableciendo como elemento central la disposición institucional (Álvarez, Chávez y Hermosilla, 2008). Así también, es necesario considerar la ideología institucional del programa o centro y la flexibilidad de los equipos de trabajo en modificar sus formas de hacer (García, Monsalvez, Ramírez y Uhart, 2007), ya que son estos últimos los que deben implementar el modelo en sus procesos de intervención. Otros elementos como: las políticas de infancia, la estabilidad de los proyectos, las 
condiciones laborales, las propias demandas y características de la población, así como el financiamiento de los programas influyen en la incorporación de la perspectiva (Demers y Zambrano, 2006).

La residencia familiar a la que se refiere esta investigación-acción, es una residencia de baja cobertura cuyo propósito es favorecer la protección integral de niñas y adolescentes gravemente vulneradas en sus derechos y que, por medida judicial, requieran ser separadas temporalmente de su medio familiar, a través de la satisfacción de sus necesidades fundamentales de subsistencia, protección y participación. La residencia atiende a 30 niñas y adolescentes entre 6 y 17 años de edad, que han ingresado con medida de protección de tribunales de familia y de competencia mixta, siendo las principales causales de ingreso: inhabilidad de uno o ambos padres, víctima de abuso sexual, testigo de violencia intrafamiliar y víctima de negligencia parental.

En esta residencia, se desarrolló la iniciativa que buscó generar un proceso de incorporación del enfoque psicoeducativo a la gestión educativa. A partir de esta experiencia, el presente estudio se ha propuesto como objetivo: identificar los elementos necesarios para el proceso de incorporación del enfoque psicoeducativo a la gestión educativa una residencia familiar.

Rescatar estos elementos supone aportar evidencia en torno a la inclusión de un enfoque de trabajo con infancia y juventud en el medio local y nacional de probada utilidad en el nivel internacional y nacional (Demers y Zambrano, 2006; Dionne y Zambrano, 2008; Vizcarra y Dionne, 2008), constituyéndose en información de importancia para orientar especialmente la intervención que actualmente se desarrolla en el sistema residencial de atención a la infancia y juventud vulnerada en sus derechos.

\section{Metodología}

Para la recogida de información, se empleó la metodología de la investigación acción. Esta metodología consta de varias etapas: diagnóstico inicial, construcción de la demanda, devolución, problematización y diagnóstico consensuado, formulación del plan estratégico, ejecución del plan y evaluación.

\subsection{Participantes}

La muestra estuvo compuesta por la totalidad del equipo que trabaja directamente con las niñas y adolescentes, siendo seis personas de sexo femenino con un promedio de 38 años y con formación secundaria en cuatro casos y universitaria en dos. 


\subsection{Procedimiento}

Para la producción de datos, se han utilizado diferentes técnicas, tales como: revisión de datos secundarios, observación participante, talleres participativos y cuestionarios semiestructurados. El proceso se organizó en las siguientes etapas:

Primera etapa: Analizando nuestro quehacer. Se ejecutó un taller diagnóstico, donde se utilizó el análisis de fortalezas, oportunidades, debilidades y amenazas (FODA), el mapa de relaciones y un autodiagnóstico a los participantes.

Segunda etapa: Definiendo líneas de acción. Se desarrolló un taller participativo, puntualizando las demandas del centro y el plan de trabajo a ejecutar.

Tercera etapa: Incorporando una nueva forma de trabajo. Primero, se realizó un proceso de formación al equipo de trabajo en la perspectiva psicoeducativa, a fin de socializar el modelo de trabajo y los instrumentos prácticos tales como: (A) Instrumentos de observación y comunicación de observaciones (bitácora, registro anecdótico, autoobservación), (B) Instrumento a partir del que se sistematizan las características observadas en los niño(as) / joven: Potencial de adaptación (PAD) y (C) Instrumento a partir del cual se diseña la intervención: Potencial experiencial (PEX). Segundo, se inició un proceso de aplicación de los instrumentos prácticos en el quehacer residencial. Tercero, se trabajó en grupo de manera quincenal, evaluando el proceso y su repercusión en la práctica, a fin de mejorar las acciones desarrolladas por educadoras de trato directo.

Así también, se trabajó con un taller participativo desarrollado en dos encuentros y coordinado por un experto en la temática, externo al centro, con el objeto de complementar las acciones desarrolladas en torno a la utilización de instrumentos e incorporación del modelo psicoeducativo a la gestión residencial, donde paralelamente se realizó la evaluación del proceso.

Cuarta etapa: Evaluando el trabajo. Se caracterizó por ser un proceso permanente durante la incorporación de la perspectiva psicoeducativa, a través de la utilización de un cuestionario semiestructurado.

\subsection{Análisis de datos}

El análisis de datos fue de tipo emergente, ya que permitió ir explorando el fenómeno, flexibilizar los procesos y modificar las técnicas, según era necesario durante el proceso de la investigación (Hernández y Fernández, 2010). Por tanto, el análisis de los resultados correspondió a un análisis en progreso que favoreció la comprensión consensuada de la realidad, contrastada y depurada en conjunto con los participantes de la investigación, quienes fueron parte del fenómeno. 
En cuanto a los mecanismos utilizados para garantizar la calidad del estudio, se utilizó la triangulación por técnica y por investigador; ya que se utilizaron distintas fuentes de datos (talleres, cuestionarios y observaciones), siendo analizados por las investigadoras de forma independiente.

Por último, cabe señalar que la investigación tomó resguardos éticos, donde los participantes accedieron a participar de forma voluntaria e informados del objetivo de la investigación; concretándose mediante la firma de un consentimiento informado.

\section{Resultados}

A partir del análisis de los resultados, se han identificado los elementos necesarios para el proceso de incorporación del enfoque psicoeducativo a la gestión educativa de una residencia familiar. En este sentido, las participantes destacan el proceso de formación donde se socializó el modelo psicoeducativo como un enfoque de trabajo, concluyendo conjuntamente su viabilidad de implementación en el centro; lográndose progresivamente el reconocimiento de este modelo y validación de sus principales herramientas metodológicas propuestas. En especial, las educadoras de trato directo seńalan que es un modelo amigable, aspecto que se acentuó en relación a los logros progresivos alcanzados en el proceso; destacándose el carácter de aplicabilidad que presenta el modelo, lo que se refleja en la utilización de instrumentos propuestos y técnicas por parte de la totalidad de las educadoras que diseñaron experiencias educativas específicas con las niñas en base a sus potenciales de adaptación.

En cuanto a los elementos de formación, se resalta el procedimiento, valorizándose positivamente las etapas del proceso de incorporación de la perspectiva psicoeducativa asociadas con la metodología de investigación acción con componentes participativos. Se reconoce que la metodología favoreció la reflexión global del quehacer residencial y permitió generar consenso respecto de la necesidad de incorporar nuevas perspectivas de intervención que mejoren la gestión educativa del centro.

Como otro elemento de formación, se destaca la utilización de la vivencia compartida que re-significa el espacio de convivencia como un espacio relacional clave para generar instancias educativas. Las educadoras ponen énfasis en los encuentros permanentes que se generan en la residencia (horarios de comida, estudios, televisión, descanso y actividades recreativos) para reflexionar en torno a las interacciones desarrolladas entre los diferentes actores que se vinculan al interior del centro y cómo estos espacios favorecen 
pequeños cambios en la interacción que pueden favorecer la labor educativa con las nińas y adolescentes.

Las educadoras resaltan que, al conocer nuevas formas de operar, es necesario tener presente no solo las actitudes del educador para enfrentar determinadas situaciones, sino que, además, las acciones que tiene el centro para identificar situaciones y las formas de abordarse, planteándose la necesidad de contar con un reglamento interno de convivencia del centro que les permita tener un marco de acción, ante situaciones complejas.

La autocrítica y disposición a aprender se atribuye al proceso mismo que se plantea como intervención, puesto que, si bien se propone la incorporación de un enfoque para innovar un estilo de gestión, se trabaja también en el campo de los significados y representaciones de las personas que constituyen y que cristalizan la gestión que pretende innovar desde una acción conjunta. En este mismo proceso, se valora el lenguaje técnico que han ido incorporando paulatinamente las educadoras.

Por último, respecto al apoyo externo de especialistas, las participantes lo reconocen como un elemento esencial del proceso, puesto que se realizó un traspaso teórico conceptual del modelo psicoeducativo no solo en la aplicabilidad del trabajo residencial, sino que, además, en las experiencias personales de las participantes, resaltando ciertas características que debe presentar el apoyo externo, tales como: identificación con la temática y experiencia en intervención con infancia.

Lo anterior ha permitido a las participantes observar y explicitar dificultades asociadas a su labor en la gestión educativa de la residencia, evidenciado que, en la intervención residencial, se carecía de lineamientos base de carácter educativo, respondiendo en mayor medida a la satisfacción de las necesidades básicas con una intervención desde la vivencia personal o práctica de las educadoras de trato directo. Por otra parte, la intervención se encontraba focalizada en la reparación del daño de las niñas y adolescentes, a través de una intervención psicosocial de tipo individual familiar, a fin de restituir el derecho a vivir en familia en el menor tiempo posible, no prestando mayor atención al proceso educativo vivenciado cotidianamente en la residencia.

Por otra parte, el recurso humano, muchas veces, ha sido seleccionado de modo centralizado por la institución, poniendo énfasis en el perfil del cargo, de acuerdo a las definiciones de las orientaciones técnicas del servicio. De acuerdo al análisis de datos secundarios, el centro ha presentado una alta rotación de personal, siendo un total de veinte personas en el transcurso de los dos ańos y medio de funcionamiento, correspondiendo al 55\% de la rotación de personal al cargo de educadora de trato directo. 
En cuanto al perfil de caso, se reconoce el aumento de la complejidad en el perfil de las niñas y adolescentes que se atienden. Así también, las educadoras de trato directo refieren no contar con herramientas suficientes para atender y abordar las nuevas necesidades y problemáticas que afectan a las nińas.

La residencia tiene una filosofía institucional que tiene, a la base, valores cristianos y mantiene una estructura jerarquizada que genera un sistema de status interno. Del mismo modo, se observa un alto compromiso social con la tarea y cohesión de sus miembros, a pesar de las contingencias que ha enfrentado el centro. La gestión desarrollada es guiada por un proyecto basado en valores institucionales y normas de funcionamiento institucional, marcada por la cultura de la beneficencia y rutinas de trabajo. Así también, el contexto residencial se caracteriza por tener un alto compromiso emocional producto de las vulneraciones de las niñas, así como las carencias que enfrenta la institución para llevar a cabo la intervención.

La participación establecida al interior del centro, por lo general, ha estado restringida a las personas adultas y a lo que estas valoran, limitándose los espacios de participación de las niñas en la convivencia comunitaria. Por otro lado, las educadoras de trato directo han visualizado espacios de participación en las reuniones técnicas, pero reconocen que, en dichos espacios, han sido receptoras de información sobre su quehacer y de las situaciones de las niñas. A partir de ello, se genera una baja articulación entre las educadoras y profesionales, relegando su rol a los cuidados y contención de las niñas. Respecto a las características de la participación, se puede decir que existen dinámicas de compañerismo y solidaridad, pero se aprecian pautas relacionales conflictivas entre las niñas marcadas por sus intereses individuales y etapas evolutivas, lo que desarrolla liderazgos que generan subgrupos al interior del centro y que son mediados por otros actores que participan en esta dinámica.

Por último, la vinculación de la residencia con el medio ha favorecido la articulación intersectorial que permite garantizar las necesidades en salud, educativas, deportivas, recreativas y de esparcimiento de las niñas, lo que se reconoce como un facilitador en el proceso de intervención residencial.

De esta forma, es posible señalar que la incorporación del enfoque psicoeducativo no solo depende de una adecuada socialización y formación del equipo de trabajo, pues además requiere de elementos contextuales e internos que le den sentido y movilicen las estructuras internas de los programas.

En cuanto a los elementos contextuales, se hace referencia a los lineamientos institucionales del Servicio Nacional de Menores (Sename), entidad pública que financia las instituciones de residencia familiar y donde ha prevaleciendo la práctica donde cada institución debe concursar por el financiamiento 
periódicamente, lo cual constituye una contingencia permanente que deben enfrentar las residencias en la actualidad y, que impacta en los diferentes operadores sociales y en el clima laboral del centro, poniendo en riesgo la continuidad de los procesos de intervención y estabilidad del programa, situación que operó, en el proceso de investigación-acción, como un importante distractor generando tensión en el ambiente subjetivo.

La ideología institucional, la infraestructura, el recurso humano y la vinculación con el medio resaltan como otros elementos de contexto que favorecen la incorporación de la perspectiva psicoeducativa como una herramienta metodológica de intervención que potencia la gestión educativa del centro y determina los procesos y los objetivos de trabajo de la atención residencial.

Respecto a los elementos internos, se reconoce el liderazgo del directivo como un facilitador del proceso de innovación, ya que permite, con mayor agilidad y rapidez, instaurar una nueva forma de trabajo por la capacidad de toma de decisión, mencionando que cuando son terceras personas que quieren incorporar nuevas iniciativas se ven obstaculizadas, debido a que en ocasiones no están validados como parte del proceso de intervención o bien no existe una información general al equipo de trabajo que los valide. Sin embargo, señalan que los directivos deben tener ciertas competencias que favorezcan el cambio cultural de la organización que puedan motivar al equipo de trabajo.

La motivación es evidenciada, por parte de las educadoras de trato directo, como un elemento que permite dinamizar y catalizar el proceso, dado que, si bien se plantea y programa un cierto plan de acción a partir del proceso, la vitalidad que este tome al ejecutarse se relaciona directamente por el nivel de motivación que presenten las educadoras y equipo en general.

Otro elemento que se resalta es la valoración de la participación de los diferentes actores involucrados, como sujetos con capacidad para determinar acciones conjuntas en torno a la intervención residencial, de donde se reconoce las educadoras como protagonistas y encargadas del proceso educativo en la residencia. Sin embargo, señalan que este proceso es gradual y de formación permanente para modificar las prácticas.

Asimismo, el autocuidado del personal surge a propósito del carácter participativo de la intervención, donde las participantes se identifican como agentes activas en el mejoramiento del clima emocional, quienes valoran, además, las ventajas que les ha traído o podría traer el trabajo desde el enfoque propuesto; puesto que revalida la noción de trabajo en equipo como una forma de trabajar y operar en el espacio laboral. De ello, destacan las fortalezas de los interventores, al mismo tiempo que destaca que estos tengan la posibilidad de buscar apoyo o contención en momento que lo requieran, hecho que favorece las acciones coor- 
dinadas y opera en función de los actos que forman parte del trabajo de las demás compañeras. Lo anterior repercute en una visión positiva de la labor de cada educadora, situación que influye positivamente en la convivencia comunitaria.

Otro elemento interno que se menciona es la planificación laboral, que tiene relación con los horarios de trabajo del personal de trato directo, como de servicio, que determina la posibilidad de incorporación del modelo. De igual modo, las participantes señalan que es pertinente el sistema de turnos rotativos, pero hacen mención que debiera existir espacios de reportes y/o administrativos para la reflexión del trabajo, a fin de intercambiar información referida a posibles situaciones de crisis evidenciadas: salidas sin autorización de adolescentes o tareas pendientes a desarrollar, entre otros, así como prever contingencias, como licencias, renuncias de personal e ingresos de otras nińas.

\section{DisCUSIÓN Y CONCLUSIONES}

Los resultados derivados de la investigación permiten observar que la residencia familiar estudiada, previo al proceso de incorporación del enfoque psicoeducativo, estaba centrada en los cuidados básicos de las niñas, no reconociéndose como un centro educativo interventor para la formación integral de nińas y adolescentes, tanto para su autoconfiguración como personas, como para el desarrollo de su autonomía e inclusión social (Martínez, 2010). Asimismo, no se había adoptado un modelo de intervención que favoreciera la gestión educativa del centro, desarrollándose la intervención residencial desde la experiencia personal y/o de práctica de las educadoras de trato directo.

Tras la implementación del estudio y la progresiva incorporación del modelo psicoeducativo a la gestión educativa del centro, se responde a la necesidad de contar con un modelo de intervención en la atención residencial que aporte al mejoramiento de programas residenciales, que han de tener un alto impacto en la protección de los niños/as y adolescentes que requieren esta medida.

El proceso ha permitido al personal repensar el modelo de atención residencial y se constituyó, de esta forma, en una manera de introducir novedad en las prácticas. Esto permitió clarificar, ordenar el trabajo y desarrollar programas educativos, a través de la utilización de los diferentes instrumentos que plantea la perspectiva. Así también, al proponer un trabajo de formación a largo plazo a las educadoras, ha favorecido, a nivel afectivo, conductual y actitudinal, a una apertura al aprendizaje y mejoramiento de las prácticas a nivel individual. Igualmente, ha generado una disposición a aprender, así como su capacidad autocrítica, es decir, repensar su rol y aprender a tomar decisiones flexibles teniendo presente las circunstancias y las características de las niñas y adolescentes. 
Al repensar su rol y actuar en consecuencia, las educadoras, junto con realzar su labor, visualizando el aporte a la formación integral de las niñas, favorecen procesos de transformación social a partir de las experiencias en un espacio educativo, pues, como señala López (2012), los aspectos psicosociales de la desigualdad se construyen, aprenden y refuerzan en los espacios de socialización primaria y, por tanto, es en estos mismos espacios donde se pueden cuestionar, ampliar las miradas y movilizar procesos transformadores.

Por otra parte, la incorporación de una nueva forma de trabajo incide en la modificación del tipo de relaciones mantenidas entre las educadoras, donde se parte de una etapa en la que el trabajo era considerado en términos más bien individuales y grupales en cuanto a coordinación de labores de menor complejidad hacia un estado actual en que se valida la discusión y análisis como una forma de mejorar el propio trabajo, así como el aporte del otro en el perfeccionamiento de este. Todo ello, involucra una directa relación con un precepto básico del enfoque psicoeducativo, el cual refiere la importancia crítica de validar las capacidades y fortalezas del otro, así como de trabajar en virtud de sus potencialidades en el marco del saber hacer y saber, como una disposición personal a relacionarnos con un otro (Vizcarra y Dionne, 2008). Así también, se avanza hacia una progresiva modificación de los espacios de participación, aumentando estos paulatinamente por cuanto se trabaja sobre la validación del otro como persona con fortalezas y capacidades, con la cual es susceptible de trabajar aquellas potencialidades por medio de una intervención que atienda realmente a sus necesidades y aspectos individuales, y que, por tanto, valide y desee su expresión, participación y empoderamiento. Así mismo, se reestructuran las dinámicas de trabajo, dándose importancia a instancias de coordinación y cooperación en donde resalta el concepto de relación de ayuda, actitudes personales y trabajo en equipo.

La incorporación del enfoque psicoeducativo favorece la resignificación de la representación de la residencia, desde una casa de acogida a un espacio de carácter comunitario en que se valide y desee la participación de los distintos actores involucrados, así como la puesta en relación entre estos. La concepción de este espacio como una comunidad, se considera como otra pieza metodológica clave de intervención que complementa los otros procesos de intervención que se desarrollan en el contexto residencial (Martínez, 2010), lo que desarrolla sistemas integrados y comprensivos de atención a las niñas y adolescentes, ya que permite integrar diferentes fases de intervención, como evaluación, programación, intervención y valoración del caso, como lo propone Del-Valle (2008) en el sistema de evaluación y registro en acogimiento residencial. Esto 
minimiza, de esta forma, la improvisación de la intervención y evita la victimización e invisibilización de los niños y adolescentes (Del-Sol-Flores, 2012).

La incorporación de la perspectiva tiene un impacto sobre el desarrollo de la comunidad (residencia), puesto que resalta la utilización de la vivencia compartida, ya que resignifica el espacio de convivencia, desde un espacio cargado de rutinas y acciones orientadas al control, a un espacio de diálogo y educación entre las educadoras y las niñas que generan aprendizajes y conocimientos. De esta manera, se favorece la planificación, coordinación y gestión educativa del centro, contexto que transforma a la residencia en un espacio de convivencia educativa cualificada, de estilo participativo y democrático, donde las educadoras y las nińas son protagonistas de la acción educativa y preventiva del centro (Cruz, 2011).

Así también, se concluye que la incorporación del enfoque a la gestión educativa de la residencia está mediada por los lineamientos institucionales de la entidad financiadora que establece un sistema de financiamiento insuficiente para cubrir las necesidades de las niñas y adolescentes que requieren la medida, así como lineamientos técnicos que solo ponen énfasis en la intervención individual, familiar y/o comunitaria, sin tener una definición desde un abordaje multinivel. Igualmente, las residencias deben cumplir con mínimos arquitectónicos, un recurso humano suficiente para la atención de las niñas residentes y contar con una planificación laboral que favorezca los procesos de intervención, así como el tiempo, espacio y otros elementos que condicionan la capacidad educativa de la residencia (Kaddur, 2007; Bravo y Del Valle, 2009).

Los aportes que el enfoque psicoeducativo entregan a la innovación de la gestión educativa del centro permite hablar de un acercamiento hacia la calidad pretendida, deseada o establecida, pues la idea de calidad remite a la idea de mejora continua en los distintos procesos involucrados (RodríguezGómez y Gairín, 2015).

En síntesis, la incorporación del enfoque psicoeducativo depende de los elementos mediadores, ya que este, con su comprensión de la relación ayuda, estrategias para utilizar la vivencia compartida y las herramientas de diagnóstico, planificación y evaluación, influye positivamente en la gestión educativa del centro. Sin embargo, su incorporación requiere de un proceso sistemático y permanente en el tiempo que confluya la presencia de ciertos criterios y condiciones para su apropiación como enfoque, filosofía y metodología de intervención por parte del personal.

En cuanto a la coherencia, esta investigación permitió fundamentar empíricamente que puede potenciarse la gestión educativa de la residencia familiar, a través de la incorporación del enfoque psicoeducativo que favorece 
la construcción paulatina de un proyecto educativo y el reconocimiento de la labor educativa de las educadoras de trato directo, quienes son las principales actoras del trabajo educativo del centro.

También queda como desafío contar con procesos de selección de personal exhaustivos que permitan generar equipos de trabajo efectivos y eficaces, indagando no solo en experiencias laborales sino también en las representaciones sociales respecto de elementos claves del trabajo realizado en la residencia, tales como: vulneración de derechos, conducta disruptiva, posibilidad de cambio, disposición al aprendizaje, entre otros.

Por otro lado, se visualiza la necesidad de mantener actividades orientadas al proceso de formación, desarrollando una línea de trabajo marcado por la permanente actualización de conocimientos, así como el reforzamiento de aquellos que ya se están trabajando en materia de psicoeducación, considerando tiempos, aspectos contractuales como dotación de personal, cambio de residencia. Sin embargo, se considera que para que lo anterior pueda llevarse a cabo, previamente es imprescindible contar con lineamientos básicos para trabajar en proceso de inducción de personal, en especial de educadoras de trato directo. Otro aspecto pendiente es la elaboración de documentación de apoyo en materia de convivencia que potencie la gestión educativa del centro.

Las limitaciones de la investigación estuvieron dadas a que no se consideró la participación de los sujetos de atención de forma activa en la investigación, cuya participación fue secundaria y mediada por los otros participantes de la investigación. Esto podría implicar un sesgo en la investigación, puesto que no considera la visión de las niñas en los resultados que podría tener la incorporación de la perspectiva en la gestión educativa del centro, contexto que impacta directamente en sus procesos de intervención. Por ello, se sugiere indagar en ello en futuras investigaciones.

\section{REFERENCIAS BIBLIOGRÁFICAS}

Álvarez, K. Chávez, J. y Hermosilla C. (2008) Incorporación del enfoque psicoeducativo en la región de la Araucanía: estudio con programas de intervención con niños y jóvenes con dificultades de adaptación social. Temuco: Universidad de La Frontera.

Bravo, A. y Del Valle, J. (2009a). Crisis y revisión del acogimiento residencial. Su papel en la protección infantil. Papeles del Psicólogo, 30(1), 42-52.

Bravo, A. y Del Valle, J. (2009b). Intervención socioeducativa en acogimiento residencial. Santander, Cantabria, España: Dirección General de Políticas Sociales. 
Colque, A. y Chesta, S. (2006). Integración del modelo psicoeducativo en la intervención con jóvenes infractores de ley. Congreso Internacional de Psicoeducación. Temuco: Universidad de La Frontera.

Cruz, L. (2011). Sobre el acogimiento residencial y las condiciones socioeducativas en las que se debe desarrollar la medida. Pedagogia i Treball social. Revista de ciències socials aplicades, 2(4), 66-88. Girona: Universitat de Girona.

Del-Sol-Flores, H. (2012). Una aproximación a la intervención educativa con menores migrantes no acompañados en España: Paradojas de la inclusión social. Revista Avaliação, 17(1), 137-153.

Del-Valle, J. (2008). Manual Cantabria. Modelo de Intervención en Acogimiento Residencial. Santander, Cantabria, España: Universidad de Oviedo.

Demers, B. y Zambrano, A. (2006). Socialización e implementación del enfoque psicoeducativo en cinco experiencias de atención a la infancia y juventud en dificultades sociales en la región de la Araucanía. Congreso Internacional de Psicoeducación. Temuco: Universidad de La Frontera

Demers, B. y Zambrano, A. (2008). La utilización en el contexto de la intervención psicoeducativa. En M. Vizcarra y J. Dionne, El desafio de la intervención psicosocial en Chile: aportes desde la psicoeducación (pp. 183-214). Santiago: RIL.

Dionne, J. y Zambrano, A. (2008). Intervención con adolescentes infractores de ley. En M. Vizcarra y J. Dionne, El desafio de la intervención psicosocial en Chile: aportes desde la psicoeducación (pp. 245-274). Santiago: RIL.

Farías, A. (2010). Evolución histórica de la política de infancia en Chile. Recuperado el 30 de noviembre 2014 de http://www.sename.gov.cl/wsename/estructuras.php?name $=$ Content $\& \mathrm{pa}=$ showpage $\& \mathrm{pid}=228$

García, A., Monsalves, C., Ramírez, T. y Uhart, P. (2007). Evaluación del proceso de incorporación del enfoque psicoeducativo en tres experiencias piloto participantes en el proyecto para los jóvenes en dificultad en la Araucanía. Temuco: Universidad de La Frontera.

Guzmán, J. (2010). Crisis en el sistema de protección de niños abandonados: el rostro invisible del bicentenario. Santiago de Chile: Ciper. Recuperado el 30 de noviembre de 2014 de http://ciperchile.cl/2010/09/02/crisis-en-el-sistemade-proteccion-de-ninos-abandonados-el-rostro-invisible-del-bicentenario/

Hernández, R. y Fernández, C. (2010). Metodología de la investigación. México: McGraw-Hill.

Kaddur, H. (2007). La atención educativa en centros de acogida de menores: el caso del centro Avicena de Melilla. Revista Eticanet, IV(6), 1-7. Recuperado el 12 de junio de 2015 de http://www.ugr.es/-sevimeco/revistaeticanet/ numero6/Tesis/Hamed_Resumen.pdf 
Lázaro, S. (2009). Resiliencia, factores protectores y necesidades en nińos y adolescentes acogidos en centros de protección. Referentes para el diseño de estrategias de intervención psicoeducativa en contextos residenciales. En A. Bravo y J. Del Valle, Intervención socioeducativa en acogimiento residencial (pp. 53-73). Santander, Cantabria, Espańa: Dirección General de Políticas Sociales.

López, N. (2012). Influencia de la escuela en la formación de los factores psicosociales de la desigualdad y los procesos de transformación social. Educación, XXI(41), 41-62.

Martínez, V. (2010). Caracterización del perfil de niños, niñas y adolescentes, atendidos por los centros residenciales de SENAME. UNICEF. Recuperado el 30 de noviembre de 2014 de http://www.sename.cl/wsename/otros/ INFORME\%20FINAL_SENAME_UNICEF.pdf

Paes-Gálvez, B. (2008). Formación en psicoeducación. En M. Vizcarra y J. Dionne, El desafio de la intervención psicosocial en Chile: aportes desde la psicoeducación (pp. 367-383). Santiago: RIL.

Rodríguez-Gómez, D. y Gairín, J. (2015). Innovación, aprendizaje organizativo y gestión del conocimiento en las instituciones educativas. Educación, XXIV(46), 73-90.

Vizcarra, M. y Dionne, J. (2008). El desafío de la intervención psicosocial en Chile. Aportes desde la psicoeducación. Santiago: RIL. 